Research Article

\title{
Digital Media Application Technology of Mobile Terminals Based on Edge Computing and Virtual Reality
}

\author{
Tao Jiang \\ School of Digital Arts \& Design, Dalian Neusoft University of Information, Dalian 116023, Liaoning, China \\ Correspondence should be addressed to Tao Jiang; jiangtao@neusoft.edu.cn
}

Received 13 May 2021; Revised 7 June 2021; Accepted 24 June 2021; Published 10 July 2021

Academic Editor: Sang-Bing Tsai

Copyright ( $\odot 2021$ Tao Jiang. This is an open access article distributed under the Creative Commons Attribution License, which permits unrestricted use, distribution, and reproduction in any medium, provided the original work is properly cited.

With the maturity of the most advanced technology and virtual reality, the application of digital media technology in various fields has become more and more extensive. Digital media technology has greatly promoted the development of all classes of society. Therefore, how to develop digital media technology applications has become a major issue. This article aims to study the digital media application technology of mobile terminals based on edge computing and virtual reality. In this paper, we use edge computing and virtual reality methods to study the digital media application technology of mobile terminals. We used the SDCEN architecture and FWA in edge computing to conduct simulation experiments and compared the delay time performance of the FWA with that of the PSO-CO algorithm, WRR algorithm, and Pick-KX algorithm. The results of this paper show that it can effectively reduce the response delay of real-time face recognition services and improve user experience. Compared with the traditional cloud computing architecture and a single MEC device, the SD-CEN network architecture based on FWA strategy has more advantages. The latency of the FWA is increased by $61 \%, 46 \%$, and $17 \%$, respectively, compared with that of the WRR, Pick$\mathrm{KX}$, and PSO-CO algorithms.

\section{Introduction}

1.1. Background of Topic Selection. As we all know, the global economic development of this century has entered a period of acceleration, and human exchange activities have gradually met the conditions for reform and transformation. After several changes, traditional mobile terminals have been guided by various emerging technologies and have undergone various optimizations, conversions, and integrations. In an environment that is eager to seek innovation and change, emerging media are more effective than traditional mobile devices. As far as the terminal is concerned, it is undeniable that it is stimulation and promotion time and time again. It can react quickly and be renewed after being stimulated. The advancement of technology has promoted the rapid development and application of digital media technology, and the gradual development of computer information technology has also made the development of digital media art a new way of technological innovation. Using digital media technology in mobile terminals, on the one hand, can make people's senses fully enjoy art; on the other hand, it can increase the amount of information dissemination and improve the effectiveness of dissemination. The combination of digital technology and mobile terminals has contributed to the development of mobile terminals and provided more space.

1.2. Significance of the Research. The reform of mobile terminals will definitely change people's lifestyles. This article hopes to conduct an in-depth study on the integration of multimedia technology and mobile terminals in order to summarize the correct design methods and lay a good foundation for future designs. In mobile terminals, actively exploring the application and development of digital technology is an urgent issue for all designers in today's society. In the context of the rapid development of digital technology, an integrated design system for screen design can be formed through the analysis, processing, and practice of screen design. Specifically, the core is the appearance of the theme, the support is digital technology and digital culture, and the pioneer is art and design. Digital media is one of the 
most basic drawing methods in screen design. The use of digital media technology in mobile terminals can not only improve the overall quality of the mobile terminal but also improve communication efficiency and technology. It realizes the effective combination of art and digital technology. In recent years, digital media technology has been widely used in various fields, and artists have applied it to their creations.

\subsection{Related Work on Digital Media Application Technology for} Mobile Terminals Based on Edge Computing and Virtual Reality. This study elaborated on mobile terminals and digital media technologies and summarized the specific impact of digital media technologies on mobile terminals. We can see new changes and new trends in the development of mobile terminals in the future. Huang et al. have studied the allocation of wireless resources and computing resources in multiuser solutions in pen edge computing, but this article does not discuss distributed computing of multiple computing devices [1]. Intharawijitr et al. have studied the application of face recognition in the Internet of Things based on edge computing, but this article is only for reference. It is proposed to uninstall part of the computational work of facial recognition applications at edge nodes, and no specific computational offloading and delay models are provided [2]. Liu et al. took the face recognition application as an example. By counting down the time between the user equipment and the edge computing server on the extreme network, it optimizes the MEC computing download strategy, verifies the effectiveness of the strategy, and determines when to download the user's computing tasks to the MEC server. However, this article did not optimize the computer uninstallation strategy on multiple MEC devices [3].

\subsection{Innovation Points of This Research.}

(1) Digital technology promotes mobile terminals, and its diversity has changed the form of mobile terminals to a certain extent

(2) In the development of digital media technology, edge computing can speed up the processing and transmission of data and reduce delays

\section{Research Methods of Digital Media Application Technology for Mobile Terminals Based on Edge Computing and Virtual Reality}

2.1. Overview of Virtual Reality Technology. Before this century, virtual reality technology was still in the exploratory stage. It only existed in certain military and academic concepts. Until the beginning of this century, with the continuous development of computer technology and sensor technology, virtual reality technology provided users with a variety of real-time physical and intuitive perceptions, such as vision, hearing, and touch, and realized physical communication between users and systems [4]. It has made it from the laboratory to the practical stage and is widely used in various fields of human life, forming a state of continuous development [5]. This type of interaction and perception method not only improves the efficiency of the system but also facilitates the user's operation. Virtual reality technology is widely regarded as having special functions of immersion, interactivity, and imagination [6].

It can be seen that virtual reality technology is a technology that uses a computer to create a virtual environment and uses the user's senses in various ways to immerse yourself in the environment to achieve natural interaction between the user and the environment [7]. Virtual reality technology allows users to use human physical skills to explore or manipulate objects in the virtual world [8].

2.2. Virtual Reality Scene Modeling Technology. The virtual reality system can only improve the perceived authenticity of the virtual reality system by creating virtual scenes that can be faked and real. The key part of it is to create a virtual model, then add sensors and stereo screens on top of it, and perform real-time rendering to complete the final construction of the virtual scene [9].

2.2.1. Geometric Modeling Technology. Geometric modeling technology is the most widely used technology for constructing virtual scenes. Combining mathematical models such as planes, curved surfaces, or spheres to adjust the outline of the virtual scene, adjust the camera to capture different roaming paths at different free angles, and add texture mapping and lighting simulation and finally render a virtual scene [10].

2.2.2. Physical Modeling Technology. The virtual model is realistic in the virtual reality system. For example, when an object is subjected to an external force, it will move naturally or collide with solid materials to produce a collision instead of passing through. Therefore, physical modeling is a further development direction than geometric modeling, that is, the physical characteristics of the real world are added to the virtual model during the modeling process. Virtual reality system modeling technology based on physical modeling methods is called physical modeling technology [11].

2.2.3. Behavioral Modeling Technology. In order to build a virtual reality system that can realistically simulate the real world, geometric modeling technology, physical modeling technology, and behavioral modeling technology must be combined [12]. In the virtual reality application environment, the virtual model is usually required to have autonomous intelligence in order to be able to interact with the user. Behavior modeling technology is based on geometric modeling technology and is responsible for describing the motion and behavior of objects. It gives the behavior and reaction of the objects in the virtual reality system, makes the virtual world full of vitality, and truly embodies the advanced nature of the virtual reality system [13]. 
2.3. Network Architecture of Edge Computing Technology. In the network architecture of edge computing technology, the capabilities of computing, storage, and business services are all immersed in edge computing technology equipment at the edge of the network. Terminal equipment can offload services at network nodes for local processing, thereby meeting the requirements of $5 \mathrm{G}$ networks. In addition, the local editing of the service can make the service closer to the user and the wireless network so that the user's current use and perception of the network location, network load, and wireless resources have effectively improved the user experience [15]. In the 5G network, the basic edge computing technology architecture is shown in Figure 1.

\subsection{Features of Edge Computing}

2.4.1. Low Latency. Different from traditional cloud computing platforms, edge computing devices are deployed at the edge of the network and provide services for users near the terminal [16]. Compared with sending data to a remote cloud computing platform for processing, it can significantly reduce time delay, save network bandwidth, and improve user experience. In addition, because the edge computing equipment is closer to the user side, it can more easily retrieve user information, thereby providing the latest network support for user data collection and data analysis [17].

2.4.2. High Bandwidth. Because edge computing devices are closer to the user side and can process data locally, there is no need to upload all data and information to the cloud. This can reduce the transmission pressure of the core network, save network bandwidth, and increase the value of network transmission [18].

2.4.3. Wide Geographical Distribution. Contrary to the central development of cloud computing in a stable location, edge computing devices are deployed at the edge of the network in a distributed manner, and services and applications can be widely distributed and deployed at the end of the computing network according to user needs [19].

2.4.4. Location Awareness. In the edge computing architecture, local services created from portable terminal devices can directly request edge computing devices for processing and can perceive the location information of each terminal device through the edge computing platform wireless network information, thereby effectively supporting locationbased service [20].

\subsubsection{Obtaining Real-Time Network Information.} Applications and services can obtain network performance information, such as wireless environment and network performance statistics, through the edge of the computer network. In addition, on this basis, users are provided with more accurate services [21].

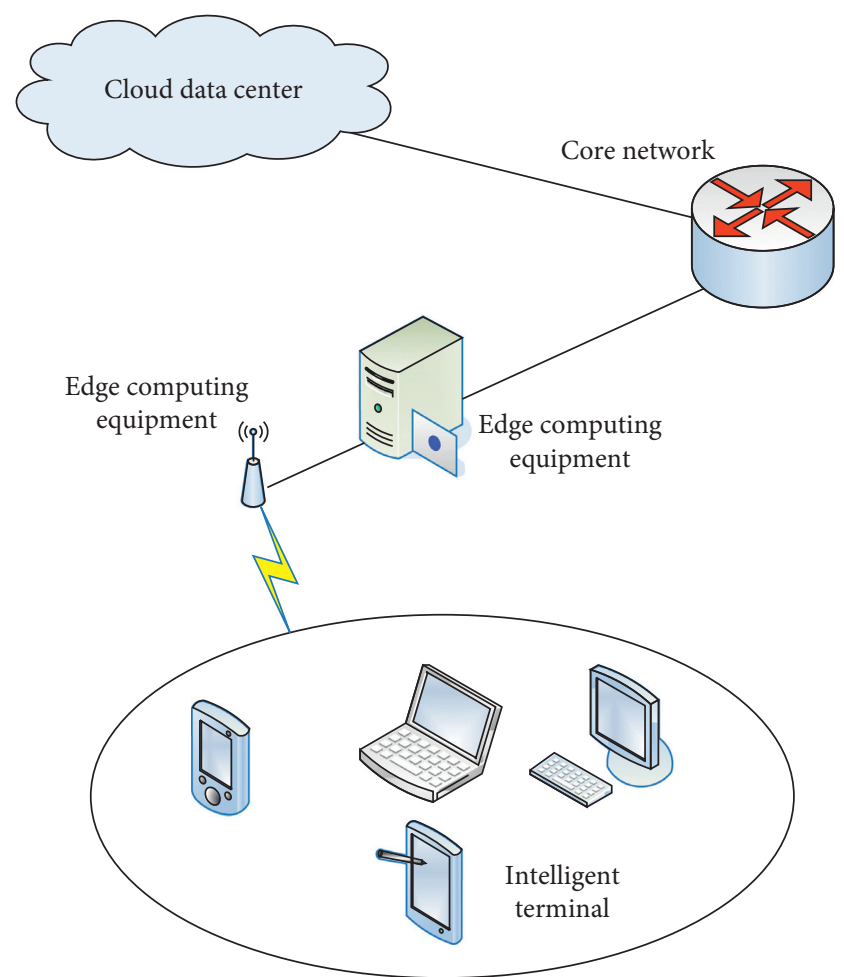

FIGURE 1: The basic network architecture of edge computing in 5G networks.

2.5. Mobile Terminal Module. A mobile terminal (MT) consists of a terminal device and a user identification module to form a small portable radio station. Its main functions include voice information coding, communication channel coding, transmission information encryption, transmission information configuration/demodulation, and antenna information transmission and reception [22]. The mobile terminal module is mainly divided into GSM module, CDMA module, and LTE module, and each module has forward compatibility. The user ID module is a mobile terminal ID card that stores user information to identify the user network. Multimedia functions have been added to CDMA and LTE drivers, and system performance has been significantly improved [14]. SIM7100CE is a mobile terminal unit based on the Qualcomm MDM9615 multimode LTE platform. It supports multiple areas, has very powerful processing capabilities, and uses $3.2 \mathrm{~V} \sim 3.6 \mathrm{~V}$ DC voltage power supply [22]. Its internal structure is shown in Figure 2.

2.6. Delay Theory Model in SD-CEN Architecture. Since the computing power of a single edge computing device is relatively weak, how to combine multiple edge computing devices to process services in a distributed manner, how to solve the best job sharing rate based on the computer and communication functions of edge computing devices, and how to use the proportion of subtasks and offload the distributed parallel computing on each MEC device have become urgent issues in the SD-CEN architecture. In order to solve the above problems, this document first models the 


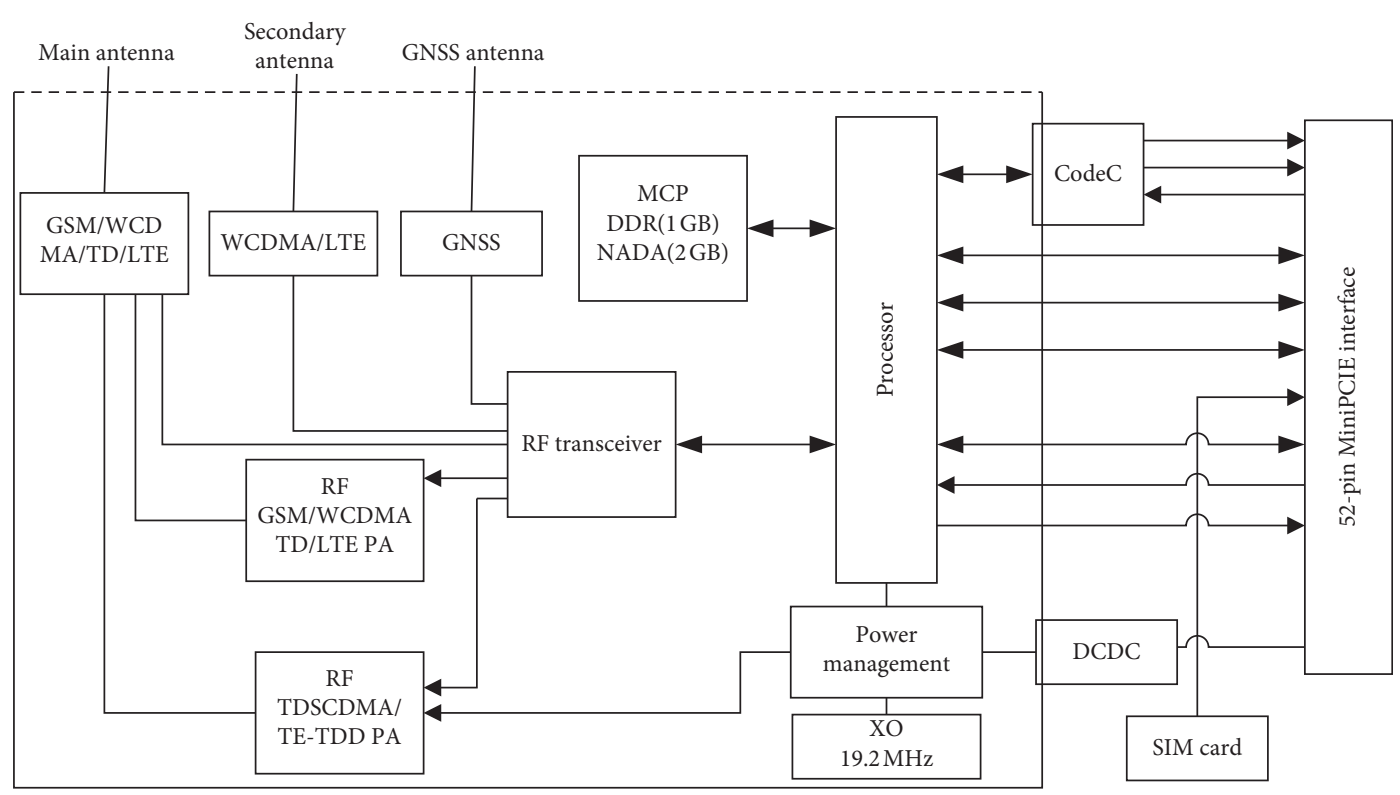

Figure 2: SIM7100CE internal structure.

service response delay in the SD-CEN architecture based on integrated communication and computer technology [23]. In this article, the completion of communication and calculation is defined as the logical separation of tasks considering the IT and communication functions of the device. In the real-time facial recognition application scenario of mobile devices, a software-defined cloud computing architecture composed of $k$ MEC devices is considered and the network topology is shown in Figure 3.

In the distributed computing process, the service response delay is equal to the maximum processing delay of all subtasks. Therefore, the total service response delay of the identification service in the SD-CEN network architecture is expressed as follows:

$$
t=\max \left\{\frac{\alpha_{i} \text { Task }}{d_{v_{i}}}+W_{v_{i}, v_{j}} m_{v_{i}, v_{j}}\right\}+\frac{\text { Task }_{\text {pre }}}{d_{d}}+W_{v_{i}, d} .
$$

Among them, $\left(\alpha_{i}\right.$ Task $\left./ d_{v_{i}}\right)$ represents the calculation time of the Task $k_{i}$ subtask on the MEC $v_{i}$ device, $W_{v_{i}, v_{j}}$ represents the communication delay between edge computing $v_{i}$ devices and sending data to the edge computing device, and $m_{v_{i}, v_{j}}$ represents whether there is a subdistribution relationship between the edge computing $v_{i}$ device and $v_{j}$. When $m_{v_{i}, v_{j}}=1$, it means that there is a secondary item allocation relationship; when $m_{v_{i}, v_{j}}=0$, it means that there is no secondary item allocation relationship. (Task $\mathrm{pre} / d_{d}$ ) represents the time delay for mapping and identifying the preprocessed feature information in the cloud, and $w_{v_{i}, c}$ represents the communication delay for sending the preprocessed feature information to the cloud server.

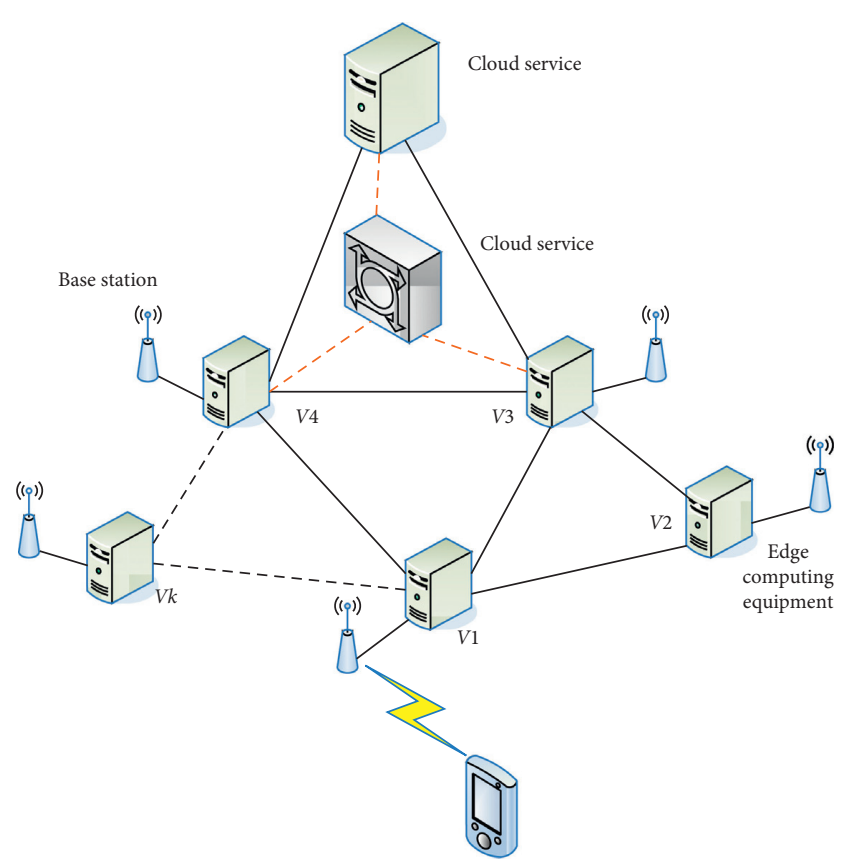

FIGURE 3: Mobile face recognition application SD-CEN network topology diagram.

In order to optimize the service response delay and achieve the goal of minimum service response delay, a set of optimal $\alpha_{i}$ job allocation coefficients are needed to minimize the delay $t$ in formula (1). In summary, in the real-time face recognition scenario, the SD-CFN network architecture service response delay can be expressed as 


$$
\begin{aligned}
& \min \max \left\{\frac{\alpha_{i} \text { Task }}{d_{v_{i}}}+W_{v_{i}, v_{j}} m_{v_{i}, v_{j}}\right\}+\frac{\text { Task }_{\mathrm{pre}}}{d_{d}}+W_{v_{i}, d} \quad i, j=1,2, \ldots, k, \\
& \text { s.t. } m_{v_{i}, v_{j}}=\left\{\begin{array}{ll}
1 & \alpha_{i} \neq 0 \\
0 & \alpha_{i}=0
\end{array},\right. \\
& \sum_{i=1}^{k} \alpha_{i}=1 .
\end{aligned}
$$

2.7. Edge Computing Optimization Delay Computing Offloading Strategy Based on Its Integration and Communication Resources. Assuming that the total task received by the edge computing $v_{1}$ device can be obtained by formula (1), the total service delay $t$ can be expressed as follows:

$$
\begin{aligned}
& t(\mathrm{TA})=\max \left\{\frac{\text { Task }_{1}}{d_{v_{1}}}+W_{v_{1}, v_{1}} m_{v_{1}, v_{1}}, \ldots, \frac{\mathrm{Task}_{k}}{d_{v_{k}}}+W_{v_{1}, v_{k}} m_{v_{1}, v_{k}}\right\}+\frac{\text { Task }_{\text {pre }}}{d_{d}}+W_{v_{1}, d} . \\
& \text { the } \alpha_{i} \text { task allocation factor can be } \\
& \text { lution of the vector TA, so formula (3) } \\
& \text { following optimization problem: } \\
& \ln \{t(\mathrm{TA})\}, \quad \mathrm{TA} \in I, \quad S_{i}=Q \times \frac{t\left(\mathrm{TA}_{i}\right)-t_{\text {min }}+\varepsilon}{\sum_{i=1}^{N}\left(t\left(\mathrm{TA}_{i}\right)-t_{\min }\right)+\varepsilon}, \\
& \sum_{i=1}^{N}\left(t_{\max }-t\left(\mathrm{TA}_{i}\right)\right)+\varepsilon
\end{aligned}
$$

Among them, $A$ is a constant that controls the maximum explosion radius and $Q$ is a constant that controls the maximum number of explosion sparks. $t_{\max }$ represents the maximum physical value of fireworks in the current firework population, namely, $t_{\max }=\max \left\{t\left(\mathrm{TA}_{i}\right)\right\} . t_{\min }$ represents the minimum physical state value of the firework in the current firework population, namely, $t_{\min }=\min \left\{t\left(\mathrm{TA}_{i}\right)\right\}$, and $\varepsilon$ is the minimum value of the machine that avoids division by zero. In formula (8), in order to avoid the influence caused by too high and too low physical values, let $a$ and $b$ be constants. Therefore, it is necessary to limit the number of sparks produced by each firework as follows:

$$
\widehat{S}_{i}= \begin{cases}\operatorname{round}(a * Q), & S_{i}<a M, \\ \operatorname{round}(b * Q), & S_{i}>b M, a<b<1, \\ \operatorname{round}\left(S_{i}\right) . & \end{cases}
$$

The explosion sparks are generated based on the calculated number of $S_{i}$ explosion sparks and $A_{i}$ explosion radius. When an explosion occurs, there will be a position shift within the explosion radius $\left[-A_{i}, A_{i}\right]$, which will be added to the $z$ dimension randomly selected by the $\mathrm{TA}_{i}$ firework, thereby generating a new explosion spark. The calculation of the selected size $z$ is as follows:

$$
z=\operatorname{round}(e * U(0,1)) \text {. }
$$

The selected dimension $z$ forms the set zs. Therefore, the explosion spark $\mathrm{A} \widehat{T}_{i}$ generated on the $k$ th dimension in the set $\mathrm{zs}$ is expressed as follows: 


$$
\mathrm{A}_{i}=\mathrm{TA}_{i k}+A_{i} \times U(-1,1), \quad k \in \mathrm{zs} .
$$

If the return size of the $\mathrm{A} \widehat{T}_{i}$ explosion spark exceeds the possible solution space range, the value of this size will be updated according to the following formula:

$$
\mathrm{A} \widehat{T}_{i}=\mathrm{TA}_{\mathrm{LB}, k}+\left|\mathrm{A} \widehat{T}_{i k}\right| \bmod \left(\mathrm{TA}_{\mathrm{UB}, k}-\mathrm{TA}_{\mathrm{LB}, k}\right) .
$$

In order to increase the diversity of the total number of sparks, Gaussian mutation sparks must be created in the firework algorithm. In the process of generating Gaussian sparks, a random $\mathrm{Mg}$ is first selected, and then the $z$ dimension is selected for each firework in the $\mathrm{Mg}$ firework to execute the Gaussian variation function according to formula (10). The calculation of the $k$ th dimension of the generated Gaussian mutation spark TA is as follows:

$$
\mathrm{TA}_{i k}{ }^{\prime}=\mathrm{TA}_{i k} \times N(1,1) .
$$

2.8. Concept of Digital Media Technology. The main body of digital media is information technology and digital technology [24]. It is a comprehensive discipline that uses information and communication technology in the fields of culture, business, art, education, and management. The source of the theory is mass communication, and the guiding method is contemporary art. In digital media technology, network communication technology, digital art, digital audio, media interaction, computer graphics, 2D animation, 3D animation, digital video and audio editing, and other technologies and innovative links are shown in a whole new way, as shown in Figure 4.

Digital media is widely used in industries such as film, television, publishing, games, news, and entertainment. It also includes Internet companies, TV stations, and other units. Specifically, it includes cartoons, online games, mobile games, digital TV and movies, digital publishing and education, and mobile TV. According to the latest research report, in 2008, digital media revenue surpassed the film industry for the first time. With the development of the media industry, the growth rate of traditional channel revenue has begun to slow down, and digital media revenue has become the main growth point of the industry, providing new growth opportunities for digital media.

\section{Experiments on Digital Media Application Technology Research of Mobile Terminals Based on Edge Computing and Virtual Reality}

3.1. Simulation Experiment. This section compares the SDCEN architecture with the traditional cloud computing architecture and a single MEC device, which delays performance when processing real-time face recognition services. It is verified that the SD-CEN architecture based on the FWA to calculate the workload download strategy can effectively reduce the service response delay in real-time face recognition scenarios, and the effectiveness of the algorithmbased computer workload offloading strategy is verified.

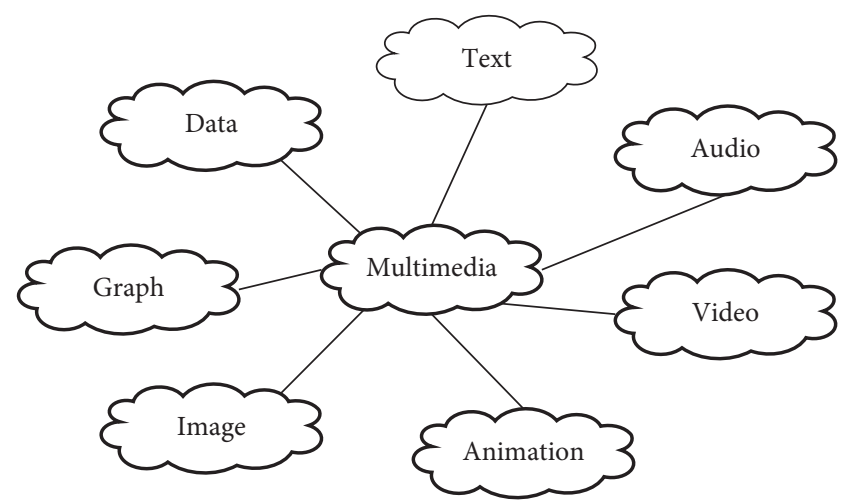

FIGURE 4: Multimedia and common elements.

In the simulation, the simulation platform uses MATLAB. The number of edge computing devices is set to 10 at the computing edge level. In the actual network environment, the functions of each edge computing device are different, and the data transmission speed and communication delay between each device are also different. The delayed communication between devices includes uplink communication delay and downlink link communication delay and other delays. Other delays mainly include data distribution delay and queue delay. Table 1 lists the relevant parameters of the SD-CEN network. The basic configuration of the FWA in the simulation is as follows: the total number of fireworks $N=40$, the maximum explosion radius $A=20$, the maximum number of explosion sparks $Q=60$, the constant $a=0.06$, the constant $b=0.9$, and the number of Gaussian sparks of the variant $\mathrm{Mg}=30$. The bandwidth of the computing power data and the connection of the MEC equipment are adjusted by reference.

\subsection{Application of Digital Media Technology}

3.2.1. Widescreen Projection Technology. In order for the audience to better understand the content to be watched, dynamic audio and video are gradually used to display static graphics in the design, especially animations, videos, and movies. This can not only present images and text in front of the audience more vividly but also provide the audience with more information. For example, even if the screen space is small, using animation to improve screen performance can also provide the public with a better information platform. In the last century, dynamic sound and video have been introduced into the display screen [25]. They have independent access areas and can also be combined in a specific wall, which is useful for publicity and decoration. Generally, exhibitions in large shopping malls produce strong visual impact. The display unit of the widescreen TV projector is shown in Table 2.

3.2.2. Multimedia Display Technology. In the information age, multimedia technology is developing rapidly. The unique video and audio effects of the audio-visual equipment can strongly display the event and leave a deep impression on the visitors [26]. Interactive multimedia technology not only enables visitors to fully understand 
TABLE 1: Relevant parameters of the SD-CEN network architecture.

\begin{tabular}{|c|c|c|c|c|c|c|c|c|c|c|c|}
\hline Parameter type & $d$ & $v_{1}$ & $v_{2}$ & $v_{3}$ & $v_{4}$ & $v_{5}$ & $v_{6}$ & $v_{7}$ & $v_{8}$ & $v_{9}$ & $v_{10}$ \\
\hline Calculation speed $(\mathrm{GHz})$ & 100 & 8 & 8.4 & 7.1 & 9.2 & 9.6 & 7.8 & 8.1 & 9.3 & 10 & 7.9 \\
\hline Uplink bandwidth (Mbps) & 5 & 80 & 64 & 73 & 86 & 9.6 & 80 & 88 & 75 & 68 & 72 \\
\hline Downlink bandwidth (Mbps) & 6 & 88 & 93 & 85 & 101 & 82 & 94 & 100 & 90 & 84 & 94 \\
\hline Other delays (ms) & 10 & 1 & 1.2 & 1.1 & 1.1 & 1.4 & 1.2 & 1.1 & 1.2 & 1.3 & 1.3 \\
\hline
\end{tabular}

TABLE 2: The display unit of the widescreen TV projector.

\begin{tabular}{lccc}
\hline & Liquid crystal & Rear projection & Plasma \\
\hline Display technology & $\begin{array}{c}\text { Connect individual pixels with } \\
\text { thin-film transistors }\end{array}$ & $\begin{array}{c}\text { Projector + rear projection screen } \\
\text { Brightness }\end{array}$ & $\begin{array}{c}\text { Low-pressure ultraviolet and variable } \\
\text { frequency wide pulse shock phosphor }\end{array}$ \\
Resolution & $1000 \mathrm{CD} / \mathrm{MM}$ & four corners $<250-300 \mathrm{CD} / \mathrm{MM}$ & $1000 \mathrm{CD} / \mathrm{MM}$ \\
Screen size & $1920 * 1080$ or $1366 * 78$ & $1024 * 768$ & $852 * 480$ \\
Image patchwork & 40 inch, 46 inch & 50 inch, 60 inch, 67 inch & Up to 42 inches \\
\hline
\end{tabular}

product information but also enables them to experience the sudden changes brought about by the showroom. The technologies used in the space exhibited at the Shanghai World Expo are holographic images and air projection systems, which account for more than $60 \%$ of the space. This is the most familiar projection method in the world, as shown in Figure 5.

3.2.3. Holographic Technology. The so-called holographic technology mainly originated from laser technology after World War II. The working principle of this technology is to divide the laser into two beams to illuminate the object and the projection film, respectively, and record the object with a special film under the illumination of a halogen lamp. Next, map it to the volume image. If you move the viewpoint appropriately, you can still see the side of the object.

3.2.4. Virtual Reality Technology. The so-called virtual reality technology refers to an interactive virtual environment, which integrates the senses of vision, hearing, and touch through the integration of information and image technology, electronic sensor settings, artificial intelligence, and sensory monitoring. In this pseudospace, the user can interact with the computer through the screen, clothes, gloves, and other devices placed in the data head to obtain a real sensory experience. Electronic games can also reflect the advantages of digital multimedia art. From a specific perspective, video games can create real scenes and sound effects and can make people feel the experience of virtual reality. The details are shown in Figure 6.

\section{Digital Media Application Technology of Mobile Terminals Based on Edge Computing and Virtual Reality}

4.1. Delay Performance Analysis of SD-CEN Architecture Based on FWA. The simulation compares the SD-CEN network architecture based on the FWA computing download strategy with the traditional cloud computing network architecture and the hysteresis performance of a single edge computing device. In the simulation, a single edge computing device selects the received MEC device $v_{1}$. The simulation result is shown in Figure 7.

4.2. Delay Performance Comparison between FWA and Various Workload Optimization Algorithms. In order to verify that the FWA can effectively reduce the service response delay in the SD-CEN network architecture, the simulation in this section will compare the delay time performance of the algorithm with that of the PSO-CO algorithm, the WRR algorithm, and the Pick-KX algorithm. The simulation results are shown in Figure 8.

Figure 5 shows the delay performance simulation results of all four algorithms. It can be seen from the figure that when the number of user requests is small, the difference between the latency of the four algorithms is very small. As the number of user requests increases, compared with the other three algorithms, the FWA has better latency performance. In the FWA, the more adaptable fireworks can produce sparks in a smaller area and have a powerful local search function. Fireworks with low fitness will produce sparks in a large area and have specific global search capabilities. WRR algorithm and Pick-KX algorithm are used to calculate unloading volume without considering communication delay and PSO-CO algorithm is easy to fall into local optimum, so the FWA can be used to obtain a better unloading strategy to effectively reduce the time lag of business response. When the number of user requests is 200, compared with the WRR, Pick-KX, and PSO-CO algorithms, the delay performance of the FWA is improved by $61 \%$, $46 \%$, and $17 \%$, respectively. Therefore, the application of the FWA in the SD-CEN architecture can effectively reduce the response delay of real-time face recognition services.

4.3. Impact of Cloud Server Uplink Bandwidth on the Delay Performance of SD-CEN Network Architecture. Since the connection bandwidth directly affects the data 

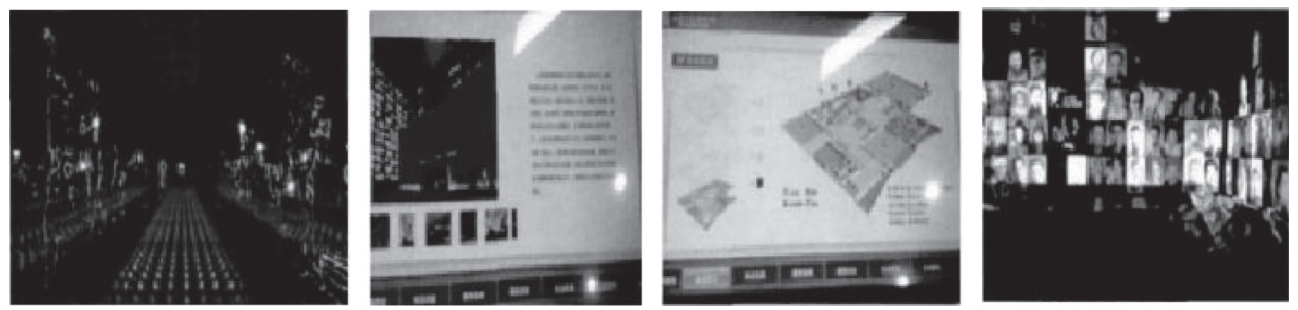

FIGURE 5: Multimedia technology used in film museums.
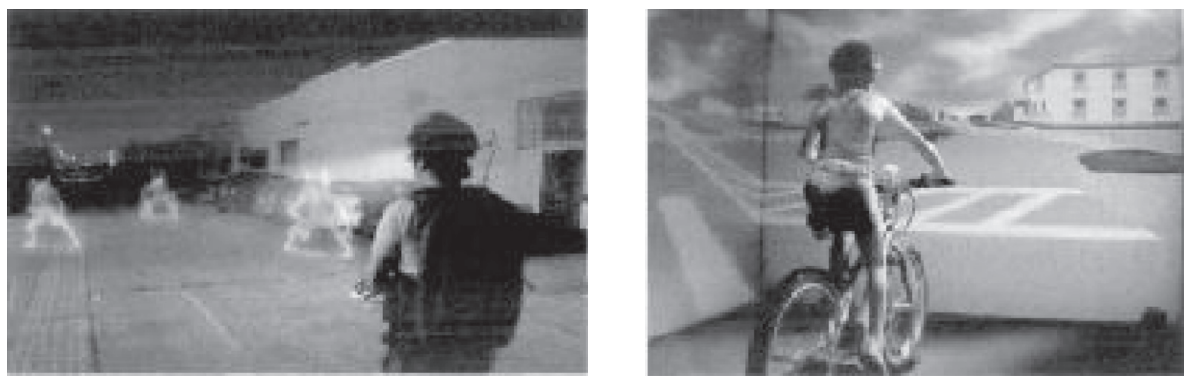

FIgURE 6: Virtual reality technology scene.

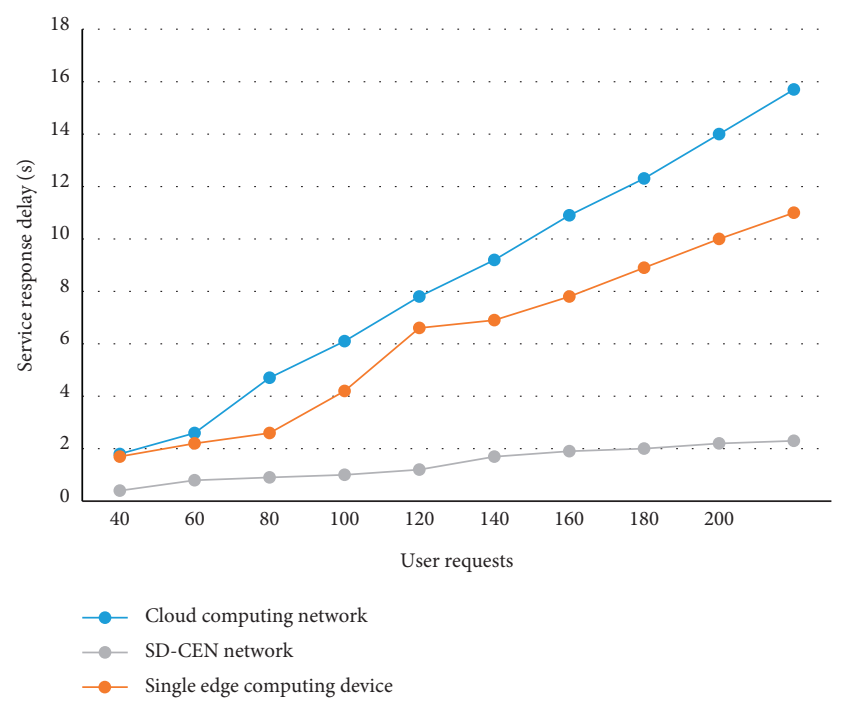

FIgURE 7: Delay performance comparison between the SD-CEN network and cloud computing network and a single edge computing device.

transmission delay, which will affect the service response delay and the actual environment, the connection bandwidth between the edge computing device and the cloud server will also change in real time. Therefore, this section studies the effect of cloud uplink server bandwidth on the structural service delay performance of the SD-CEN network system. When the number of user requests is 200 , the upstream bandwidth of the cloud server changes from $5 \mathrm{Mbps}$ to $30 \mathrm{Mbps}$. The simulation result is shown in Figure 9.

Figure 9 shows that as the link bandwidth continues to increase, the service response delay of the cloud computing

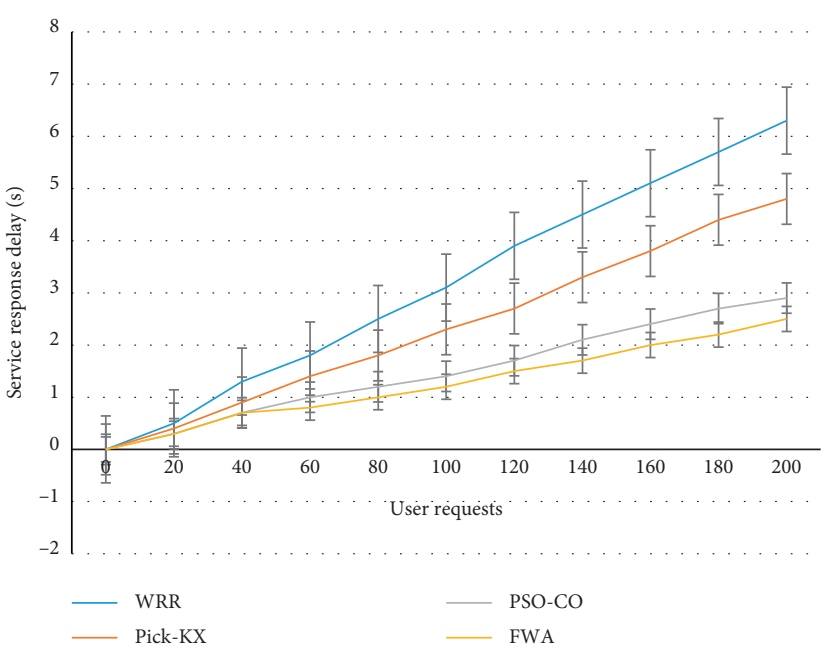

FIGURE 8: Delay performance comparison between FWA and various workload optimization algorithms.

architecture is gradually reduced, but the service response delay of the SD-CEN network architecture is only reduced by a small part. In the cloud computing architecture, the increase in link bandwidth will result in a significant reduction in data transmission delay. Therefore, the service response delay of the cloud computing architecture will increase and decrease as the bandwidth increases. In the SD$\mathrm{CEN}$ architecture, the amount of facial feature information data after preprocessing is very small. Therefore, as the link bandwidth of the cloud server increases, the delay performance of the SD-CEN network architecture will only slightly decrease. In summary, when the upstream bandwidth of the cloud server is limited, the SD-CEN network architecture has a more obvious advantage in reducing service response delay. 


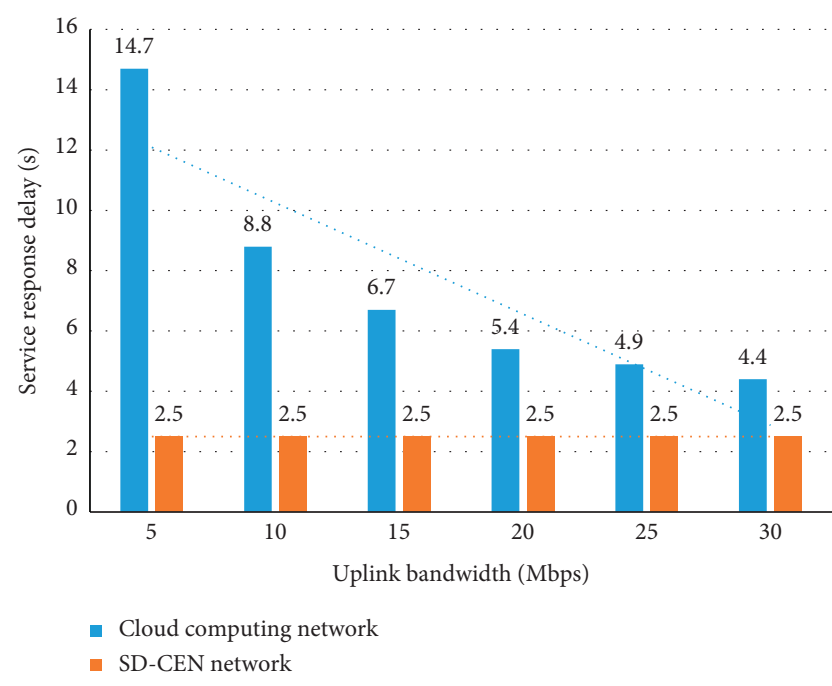

FIgURE 9: The impact of cloud server uplink bandwidth on the delay performance of the SD-CEN network architecture.

\section{Conclusion}

This study elaborated on mobile terminals and digital media technologies and summarized the specific impact of digital media technologies on mobile terminals. We have seen new changes and new trends in the development of mobile terminals in the future. Most importantly, the use of digital media technology will not change the purpose of the mobile terminal. On the contrary, it provides a more intuitive and scientific appearance for the mobile terminal. In the actual screen design process, the quality of the exhibition objects should emphasize the use of digital multimedia technology. Focusing on content, all digital media technologies are used for demonstration and maintenance projects. At the same time, designers must not only establish new ideas for traditional mobile terminals but also must put forward higher requirements for their own comprehensive quality training. The integration of digital media and mobile terminals will become closer, and digital media technology will also play a greater role in the achievements of mobile terminals. It is predicted that the future society will be science and technology, and mobile terminals will continue to develop in this direction. The relationship between mobile terminals and people's daily lives will become closer and closer, and every plan must be advanced.

\section{Data Availability}

No data were used to support this study.

\section{Conflicts of Interest}

The author declares no conflicts of interest.

\section{References}

[1] L. Huang, X. Feng, C. Zhang, L. Qian, and Y. Wu, "Deep reinforcement learning-based joint task offloading and bandwidth allocation for multi-user mobile edge computing,"
Digital Communications and Networks, vol. 5, no. 1, pp. 10-17, 2019.

[2] K. Intharawijitr, K. Iida, and H. Koga, "Simulation study of low latency network architecture using mobile edge computing," IEICE Transactions on Information and Systems, vol. E100.D, no. 5, pp. 963-972, 2017.

[3] X. Liu, J. Yu, Z. Feng, and Y. Gao, "Multi-agent reinforcement learning for resource allocation in IoT networks with edge computing," China Communications, vol. 17, no. 9, pp. 220-236, 2020.

[4] E. Ahmed, A. Ahmed, I. Yaqoob et al., "Bringing computation closer toward the user network: is edge computing the solution?" IEEE Communications Magazine, vol. 55, no. 11, pp. 138-144, 2017.

[5] E. Bastug, M. Bennis, M. Medard, and M. Debbah, "Toward interconnected virtual reality: opportunities, challenges, and enablers," IEEE Communications Magazine, vol. 55, no. 6, pp. 110-117, 2017.

[6] M. S. Elbamby, C. Perfecto, M. Bennis, and K. Doppler, "Toward low-latency and ultra-reliable virtual reality," IEEE Network, vol. 32, no. 2, pp. 78-84, 2018.

[7] D. C. Li, B.-H. Chen, C.-W. Tseng, and L.-D. Chou, "A novel genetic service function deployment management platform for edge computing," Mobile Information Systems, vol. 2020, Article ID 8830294, 22 pages, 2020.

[8] O. Oyman, R. Koenen, P. Higgs et al., "Virtual reality industry forum's view on state of the immersive media industry," SMPTE Motion Imaging Journal, vol. 128, no. 8, pp. 91-96, 2019.

[9] S. Sukhmani, M. Sadeghi, M. Erol-Kantarci, and A. El Saddik, "Edge caching and computing in 5G for mobile AR/VR and tactile internet," IEEE MultiMedia, vol. 26, no. 1, pp. 21-30, 2019.

[10] Y. Sun, Z. Chen, M. Tao et al., "Communications, caching and computing for mobile virtual reality: modeling and tradeoff," IEEE Transactions on Communications, vol. 67, no. 99, pp. 7573-7586, 2019.

[11] X. Qiao, P. Ren, S. Dustdar et al., "Web AR: a promising future for mobile augmented reality-state of the art, challenges, and insights," Proceedings of the IEEE, vol. 107, no. 4, pp. 651-666, 2019.

[12] Y. Zhang, L. Jiao, J. Yan, and X. Lin, "Dynamic service placement for virtual reality group gaming on mobile edge cloudlets," IEEE Journal on Selected Areas in Communications, vol. 37, no. 8, pp. 1881-1897, 2019.

[13] S. Jung and H. Kim, "A study on the production plan for reducing cybersickness in virtual reality game," Journal of the Korea Society of Digital Industry and Information Management, vol. 13, no. 1, pp. 113-123, 2017.

[14] A. Shakarami, M. Ghobaei-Arani, M. MaSDari et al., "A survey on the computation offloading approaches in mobile edge/cloud computing environment: a stochastic-based perspective," Journal of Grid Computing, vol. 18, no. 4, pp. 1-33, 2020.

[15] E. Li and J. Yu, "A forensic mobile application designed for both steganalysis and steganography in digital images," Electronic Imaging, vol. 6, pp. 84-89, 2017.

[16] Y. Zhao, "A model research on internet ideological and political work in colleges and universities under the background of new media," Revista de la Facultad de Ingenieria, vol. 32, no. 14, pp. 90-96, 2017.

[17] Y. Endo, K. Saito, S. Watanabe, M. Takahashi, and K. Ito, "Study of interference voltage of an implanted pacemaker by 
mobile terminals," IEEE Transactions on Electromagnetic Compatibility, vol. 58, no. 1, pp. 30-39, 2016.

[18] W. Zhu, "Digital media technology's application in digital dance," Journal of the Korea Entertainment Industry Association, vol. 12, no. 4, pp. 345-354, 2018.

[19] D. Yu, "Analysis of rural culture construction and innovation based on integration of digital media technology and paper material," Paper Asia, vol. 1, no. 9, pp. 122-125, 2018.

[20] Y. Shi, C. Feng, and Y. Shen, "Research on graphic design based on digital media," Acta Technica CSAV (Ceskoslovensk Akademie Ved), vol. 62, no. 1, pp. 391-400, 2017.

[21] H. Chen, "Research on the application of digital media art in animation control based on Maya MEL language," Acta Technica CSAV (Ceskoslovensk Akademie Ved), vol. 62, no. 1, pp. 499-507, 2017.

[22] S. Li, Q. Wang, Y. Wang et al., "Joint congestion control and resource allocation for delay-aware tasks in mobile edge computing," Wireless Communications and Mobile Computing, vol. 2021, Article ID 8897814, 16 pages, 2021.

[23] Z.-G. Liu, Y. Yang, and X.-H. Ji, "Flame detection algorithm based on a saliency detection technique and the uniform local binary pattern in the $\mathrm{YCbCr}$ color space," Signal, Image and Video Processing, vol. 10, no. 2, pp. 277-284, 2016.

[24] L. Fabisiak, "Web service usability analysis based on user preferences," Journal of Organizational and End User Computing, vol. 30, no. 4, pp. 1-13, 2018.

[25] H. Xinjue, Q. Wei, G. Tao et al., "Mobile edge assisted live streaming system for omnidirectional video," Mobile Information Systems, vol. 2019, Article ID 8487372, 15 pages, 2019.

[26] H. Kim, "Investigating the mediating role of social networking service usage on the big five personality traits and on the job satisfaction of Korean workers," Journal of Organizational and End User Computing, vol. 31, no. 1, pp. 110-123, 2019. 